\title{
Spectral response of dielectric nano-antennas in the far- and near-field regimes
}

\author{
Y. Gutiérrez, Á. I. Barreda, F. González, F. Moreno \\ Group of Optics, Department of Applied Physics, University of Cantabria, Avenida de Los \\ Castros s/n, 39005 Santander, Spain
}

\begin{abstract}
Recent studies show that the spectral behaviour of localized surface plasmon resonances (LPSRs) in metallic nanoparticles suffer from both a redshift and a broadening in the transition from the far- to the near-field regimes. An interpretation of this effect was given in terms of the evanescent and propagating components of the angular spectrum representation of the radiated field. Due to the increasing interest awakened by magnetodielectric materials as a both low-loss material option for nanotechnology applications, and also for their particular scattering properties, here we study the spectral response of a magnetodielectric nanoparticle as a basic element of a dielectric nano-antenna. This study is made by analyzing the changes suffered by the scattered electromagnetic field when propagating from the surface of this dielectric nanostructure to the far-zone in terms of propagating and evanescent plane wave components of the radiated fields.
\end{abstract}

Keywords: Magnetodielectric, radiative, non-radiative, redshift, broadening, LSPR

\section{INTRODUCTION}

Plasmonics is the branch of Nanophotonics which studies the confinement and enhancement of the local electromagnetic field produced in metal nanoparticles when electromagnetally irradiated. This effects, produced by the resonant behaviour of the electronic plasma, are known as localized surface plasmon resonances (LSPRs). This phenomenon has proven its utility in a wide range of nanotechnological fields such as optical communications, spectroscopy or medical treatments. In these applications it is critical to have the control of the frequency at which the LSPRs are produced. Usually, the position of the LSP peaks are obtained in the far-field domain $(k r>>1)$ by conventional parameters such as the scattering or extinction spectral efficiencies. However, some surface spectroscopy techniques like Surface Enhancement Raman Spectroscopy (SERS), requires of the control of the LSP peak position in the near-field regimes $(k r<1)$ in order to get their optimum efficiency. In recent studies it has been shown that for metallic particles under the dipole approximation, there is a clear redshift and broadening of the nano-antenna plasmon spectral response when it is compared in the near- and far-field. ${ }^{1-3}$ Analytical expressions for predicting these shifts have been also recently proposed. ${ }^{4}$

One of the main disadvantages of using metals for plasmonic applications is that their performance is inherently accompanied by ohmic losses. This loss mechanism leads to Joule heating of both the nanostructure and its local environment. ${ }^{5-7}$ This effect has its advantages in applications in which photothermal effects are necessary. ${ }^{8}$ However, for other applications in which matter is analyzed through surface field enhancements, this local heating can alter, or even destroy the samples. As an alternative, magnetodielectric materials (dielectrics with refractive index $m>3$, like silicon and germanium in the near infrarred (NIR)) have been recently proposed as a low-loss option at their corresponding spectral range, and also for its particular scattering properties due to the possible coherent excitations of electric and magnetic dipolar resonances. ${ }^{9,10}$

In this work, we perform a detailed study of the spectral response of a magnetodielectric nanosphere as a basic element of a dielectric nano-antenna. This research is performed by studying the changes suffered by the scattered, or emitted, electromagnetic field on propagation from the surface of nanostructure to the far-zone in terms of propagating and evanescent plane wave components of the angular spectrum representation of the

Further author information: (Send correspondence to F. Moreno)

F. Moreno.: E-mail: morenof@unican.es

Photonic and Phononic Properties of Engineered Nanostructures VI, edited by Ali Adibi, Shawn-Yu Lin, Axel Scherer, Proc. of SPIE Vol. 9756, 975628 - @ 2016 SPIE · CCC code: 0277-786X/16/\$18 - doi: 10.1117/12.2229490 
scattered fields. These components are the key concepts for the understanding of the near-field optics versus the more conventional far-field observations.

The work is organized as follows: in Section 2, we briefly review the theoretical concepts. In Section 3, we show the main results of this research. Finally, in Section 4 its main conclusions are presented.

\section{THEORETICAL BACKGROUND}

In general, the emitted field in a scattering process is composed of evanescent and propagating contributions. The evanescent waves keep attached to the scatterer surface, so their amplitude decreases exponentially as the distance from the surface increases. This non-radiative contribution was shown to be responsible for the near-field spectral red-shifts for metallic nanoparticles. ${ }^{2}$ Whereas in the far field the spectral behaviour of the metallic dipolar nano-antenna was dominated by the propagating waves (or radiative contribution), in the near-field regime the non-radiative contribution takes the lead role. The calculations of both radiative and non-radiative contribution can be achieved by the angular spectrum decomposition method of the radiated fields. For a sphere, the general solution of this problem in terms of the Mie expansion was solve by Kvien. ${ }^{11}$

The scattering geometry is shown in Fig. 1. A linearly polarized (parallel to the X axis) electromagnetic plane wave of wavelength $\lambda$ and propagating along the $\mathrm{Z}$ axis, illuminates a magnetodielectric spherical particle of radius $a$ placed in the vacuum $\left(\epsilon_{m}=1\right)$. Because we are treating with a magnetodielectric particle, its electric permittivity will be considered to be purely real $\left(\epsilon=\epsilon_{r}\right)$ such as silicon in the NIR, where the imaginary part of its electric permittivity can be neglected. The magnetic permeability will be considered to be $\mu=1$.

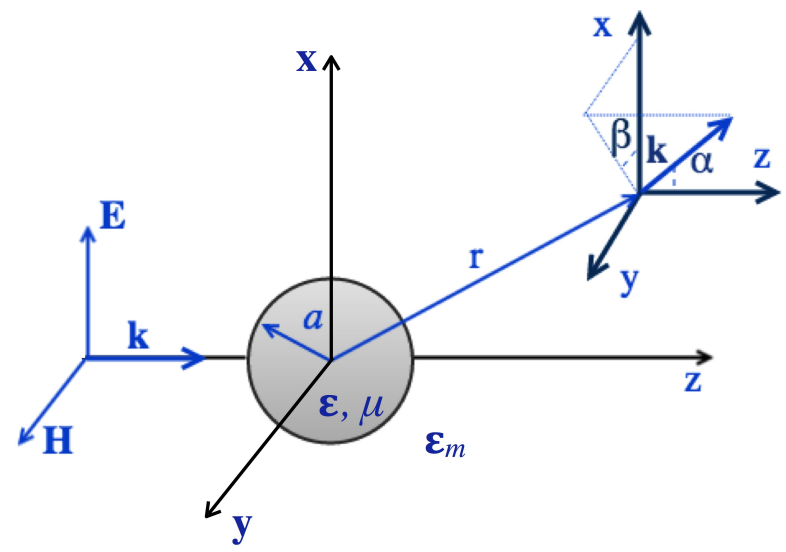

Figure 1. Scattering geometry. A magnetodielectric spherical particle is illuminated with a plane wave electromagnetic field travelling in the Z-direction and linearly polarized in the X-direction. At a point $\vec{r}$, the contribution of the plane wave with wave vector $\vec{k}(\alpha, \beta)$ to the angular spectrum of the scattered field is also represented.

The scattered electric and magnetic fields, $\vec{E}_{s}(\vec{r})$ and $\vec{H}_{s}(\vec{r})$, at a $\vec{r}$ point outside the particle can be expressed as an angular spectrum of plane waves

$$
\begin{aligned}
& \vec{E}_{s}(\vec{r})=\frac{1}{2 \pi} \int_{0}^{2 \pi} d \beta \int_{0}^{\frac{\pi}{2}-i \infty} e^{i \vec{k} \vec{r}}\left[S_{1}(\cos \alpha) \cos \beta \overrightarrow{e_{\alpha}}-S_{2}(\cos \alpha) \sin \beta \overrightarrow{e_{\beta}}\right] \sin \beta d \alpha \\
& \vec{H}_{s}(\vec{r})=\frac{1}{2 \pi} \int_{0}^{2 \pi} d \beta \int_{0}^{\frac{\pi}{2}-i \infty} e^{i \vec{k} \vec{r}}\left[S_{2}(\cos \alpha) \sin \beta \overrightarrow{e_{\alpha}}+S_{1}(\cos \alpha) \cos \beta \overrightarrow{e_{\beta}}\right] \sin \beta d \alpha
\end{aligned}
$$

where $\vec{k}$ is the wave vector whose modulus is given by $|\vec{k}|=k=\frac{2 \pi}{\lambda}$. The $\overrightarrow{e_{\alpha}}$ and $\overrightarrow{e_{\beta}}$ are the unitary vectors in the $\alpha$ direction with respect to the Z-axis, and in the azimuthal direction $\beta$ on the XY-plane, respectively. The angular spectra $S_{1}(\cos \alpha)$ and $S_{2}(\cos \alpha)$ functions in equations (1) and (2) are given by 


$$
\begin{aligned}
& S_{1}(\cos \alpha)=\sum_{n=1}^{\infty} \frac{2 n+1}{n(n+1)}\left[a_{n} \pi_{n}(\cos \alpha)+b_{n} \tau_{n}(\cos \alpha)\right] \\
& S_{2}(\cos \alpha)=\sum_{n=1}^{\infty} \frac{2 n+1}{n(n+1)}\left[a_{n} \tau_{n}(\cos \alpha)+b_{n} \pi_{n}(\cos \alpha)\right]
\end{aligned}
$$

being $\pi_{n}$ and $\tau_{n}$ the angle dependent functions from Mie scattering theory.

In our analysis, the particle's size is much smaller than the incident wavelength. As a result, only the dipolar terms in the Mie expansion $(n=1)$ will be considered. So we are treating magnetodielectric particles weighted by the Mie coefficients a1 (dipolar electric) and b1 (dipolar magnetic). Under these approximations, equations 1 and 2 can be rewritten as

$$
\begin{aligned}
\vec{E}_{s}(\vec{r})= & \frac{3 a_{1}}{4 \pi} \int_{0}^{2 \pi} d \beta \int_{0}^{\frac{\pi}{2}-i \infty} d \alpha \sin \alpha\left(\cos \beta \overrightarrow{e_{\alpha}}-\cos \alpha \sin \beta \overrightarrow{e_{\beta}}\right) e^{i \vec{k} \cdot \vec{r}}+ \\
& \frac{3 b_{1}}{4 \pi} \int_{0}^{2 \pi} d \beta \int_{0}^{\frac{\pi}{2}-i \infty} d \alpha \sin \alpha\left(\cos \alpha \cos \beta \overrightarrow{e_{\alpha}}-\sin \beta \overrightarrow{e_{\beta}}\right) e^{i \vec{k} \cdot \vec{r}} \\
\vec{H}_{s}(\vec{r})= & \frac{3 a_{1}}{4 \pi} \int_{0}^{2 \pi} d \beta \int_{0}^{\frac{\pi}{2}-i \infty} d \alpha \sin \alpha\left(\cos \alpha \sin \beta \overrightarrow{e_{\alpha}}+\cos \beta \overrightarrow{e_{\beta}}\right) e^{i \vec{k} \cdot \vec{r}}+ \\
& \frac{3 b_{1}}{4 \pi} \int_{0}^{2 \pi} d \beta \int_{0}^{\frac{\pi}{2}-i \infty} d \alpha \sin \alpha\left(\sin \beta \overrightarrow{e_{\alpha}}+\cos \alpha \cos \beta \overrightarrow{e_{\beta}}\right) e^{i \vec{k} \cdot \vec{r}}
\end{aligned}
$$

In our laboratory system, $\vec{k}=\frac{2 \pi}{\lambda}(\sin \alpha \cos \beta, \sin \alpha \sin \beta, \cos \alpha), \overrightarrow{e_{\alpha}}=(\cos \alpha \cos \beta, \cos \alpha \sin \beta,-\sin \beta)$ and $\overrightarrow{e_{\beta}}=(\sin \beta, \cos \beta, 0)$. The integration interval of the angular coordinate $\alpha$ of equations $(5)$ and $(6),\left[0, \frac{\pi}{2}-i \infty\right]$, can be divided in two parts: $\left[0, \frac{\pi}{2}\right]$ and $\left[\frac{\pi}{2}-i 0, \frac{\pi}{2}-i \infty\right]$. The intregation over the first of the previous intervals leads to the radiative contribution, $\vec{E}_{s}^{R}(\vec{r})$ and $\vec{H}_{s}^{R}(\vec{r})$, whereas the integration over second interval corresponds to the non-radiative contribution, $\vec{E}_{s}^{N-R}(\vec{r})$ and $\vec{H}_{s}^{N-R}(\vec{r}) .{ }^{12}$ Taking this into account, equations (5) and (6) can also be expressed as

$$
\begin{aligned}
& \vec{E}_{s}(\vec{r})=\vec{E}_{s}^{R}(\vec{r})+\vec{E}_{s}^{N-R}(\vec{r}) \\
& \vec{H}_{s}(\vec{r})=\vec{H}_{s}^{R}(\vec{r})+\vec{H}_{s}^{N-R}(\vec{r})
\end{aligned}
$$

The total radiated field can be expressed as those radiated by an electric dipole crossed to a magnetic one. On the one hand, the electric and magnetic fields radiated by the electric dipole, $\vec{E}_{s}^{\text {elec }}(\vec{r})$ and $\vec{H}_{s}^{\text {elec }}(\vec{r})$, are given by $^{13}$

$$
\begin{gathered}
\vec{E}_{s}^{\text {elec }}(\vec{r})=\frac{1}{4 \pi \epsilon_{0}}\left[\vec{p}\left(k^{2}-\frac{1}{r^{2}}+\frac{i k}{r}\right)+\vec{n}(\vec{n} \cdot \vec{p})\left(-k^{2}+\frac{3}{r^{2}}+\frac{3 i k}{r}\right)\right] \frac{e^{i k r}}{r} \\
\vec{H}_{s}^{\text {elec }}(\vec{r})=\frac{1}{4 \pi \sqrt{\mu_{0} \epsilon_{0}}}\left[(\vec{n} \times \vec{p})\left(k^{2}+\frac{i k}{r}\right)\right] \frac{e^{i k r}}{r}
\end{gathered}
$$

On the other, those radiated by the magnetic dipole, $\vec{E}_{s}^{\operatorname{mag}}(\vec{r})$ and $\vec{H}_{s}^{\operatorname{mag}}(\vec{r})$, are given by ${ }^{13}$

$$
\vec{E}_{s}^{m a g}(\vec{r})=-\frac{1}{4 \pi} \sqrt{\frac{\mu_{0}}{\epsilon_{0}}}\left[(\vec{n} \times \vec{m})\left(k^{2}+\frac{i k}{r}\right)\right] \frac{e^{i k r}}{r}
$$




$$
\vec{H}_{s}^{m a g}(\vec{r})=\frac{1}{4 \pi}\left[\vec{m}\left(k^{2}-\frac{1}{r^{2}}+\frac{i k}{r}\right)+\vec{n}(\vec{n} \cdot \vec{m})\left(-k^{2}+\frac{3}{r^{2}}+\frac{3 i k}{r}\right)\right] \frac{e^{i k r}}{r}
$$

where $r=|\vec{r}|$, and $\vec{n}$ is the unit vector pointing in the $\vec{r}$ direction. $\vec{p}$ and $\vec{m}$ are the electric and magnetic dipolar moments induced in the particle by the incident electric field, $\vec{E}_{i}(\vec{r})=\vec{E}_{0} e^{i\left(\overrightarrow{k_{i}} z-\omega t\right)}$ and are given by

$$
\begin{aligned}
& \vec{p}=\epsilon_{0} \alpha_{E} \vec{E}_{0} \\
& \vec{m}=\alpha_{M} \vec{H}_{0}
\end{aligned}
$$

where $\alpha_{E}$ and $\alpha_{M}$ are the electric and magnetic polarizabilities of the spherical particle. These can be expressed in terms of the Mie coefficients $a_{1}$ and $b_{1}$ as ${ }^{14}$

$$
\begin{aligned}
\alpha_{E} & =\frac{i 6 \pi a_{1}}{k^{3}} \\
\alpha_{M} & =\frac{i 6 \pi b_{1}}{k^{3}}
\end{aligned}
$$

Then, the total electric and magnetic radiated fields by a magnetodielectric sphere can be expressed as

$$
\begin{aligned}
\vec{E}_{s}(\vec{r}) & =\vec{E}_{s}^{\text {elec }}(\vec{r})+\vec{E}_{s}^{\text {mag }}(\vec{r}) \\
\vec{H}_{s}(\vec{r}) & =\vec{H}_{s}^{\text {elec }}(\vec{r})+\vec{H}_{s}^{\text {mag }}(\vec{r})
\end{aligned}
$$

Eqs. (17) and (18) provide an easy way to separate both the electric and magnetic dipolar contributions. For instance, by taking $b_{1}=0$, we are only considering the electric dipolar term, and by taking $a_{1}=0$, only the magnetic dipolar contribution is being considered. The same approach can be done with equations (5) and (6), which allow us to calculate the radiative and non-radiative contributions of both dipolar terms separately. Another interesting feature of this representation is that the Mie coefficients $a_{1}$ and $b_{1}$, that appear as a factor in every electromagnetic field contribution (see eqs. (7) and (8)), contains all the information about the morphology and optical properties of the particle. As a result, by dividing either by $a_{1}$ or $b_{1}$ the radiative and non-radiative contributions, it is possible to obtain a general law for the spectral behaviour of the electric (magnetic) dipolar terms.

In this work, we are interested in calculating the contribution of the non-radiative part to the total radiated field. The calculation of this contribution involves the integration over an imaginary interval. However, the calculation of the total and radiative contribution can be performed in a more straightforward way. Therefore, $\vec{E}_{s}^{N-R}(\vec{r})$ and $\vec{H}_{s}^{N-R}(\vec{r})$ can be expressed as

$$
\begin{aligned}
& \vec{E}_{s}^{N-R}(\vec{r})=\vec{E}_{s}(\vec{r})-\vec{E}_{s}^{R}(\vec{r}) \\
& \vec{H}_{s}^{N-R}(\vec{r})=\vec{H}_{s}(\vec{r})-\vec{H}_{s}^{R}(\vec{r})
\end{aligned}
$$

A magnitude which is experimentally interesting is the scattering intensity. This parameter is directly related with the square modulus of the electric and magnetic fields, $|\vec{E}|^{2}$ and $|\vec{H}|^{2}$. The expression for these two magnitudes can be obtained from eqs. (7) and (8), and can be written as follows

$$
\begin{aligned}
\left|\vec{E}_{s}(\vec{r})\right|^{2} & =\left|\vec{E}_{s}^{N-R}(\vec{r})\right|^{2}+\left|\vec{E}_{s}^{R}(\vec{r})\right|^{2}+2 \operatorname{Re}\left(\vec{E}_{s}^{N-R}(\vec{r})^{*} \cdot \vec{E}_{s}^{R}(\vec{r})\right) \\
\left|\vec{H}_{s}(\vec{r})\right|^{2} & =\left|\vec{H}_{s}^{N-R}(\vec{r})\right|^{2}+\left|\vec{H}_{s}^{R}(\vec{r})\right|^{2}+2 \operatorname{Re}\left(\vec{H}_{s}^{N-R}(\vec{r})^{*} \cdot \vec{H}_{s}^{R}(\vec{r})\right)
\end{aligned}
$$

As it can be seen, the intensity of the total scattered field is not just the sum of the intensities of both contributions. A interference term between the radiative and non radiative contribution has to be added too. 


\section{RESULTS}

The spectral response of a spherical magnetodielectric particle with radius $a=7,5 \mathrm{~mm}$ and $\epsilon=16$ is going to be studied over the spectral interval $40-70 \mathrm{~mm}$. Although we are treating with a particle of millimeter size, its behaviour is equivalent to those of a nanoparticle with the same optical properties in the visible spectral range. This is because of the scaling property of the Maxwell equations. The interest of working with particle sizes of several millimetres is due to experimental reasons. ${ }^{10}$

In Fig. 2 we show $\left|\vec{E}_{s}(\vec{r})\right|^{2}$ (red solid line), $\left|\vec{E}_{s}^{R}(\vec{r})\right|^{2}$ (green solid line), $\left|\vec{E}_{s}^{R}(\vec{r})\right|^{2}$ (blue solid line) and the interference term $2 \operatorname{Re}\left(\vec{E}_{s}^{N-R}(\vec{r})^{*} \cdot \vec{E}_{s}^{R}(\vec{r})\right)$ (magenta solid line) at points $\vec{r}=(a, 0,0)$ and $\vec{r}=(0,0, a)$, where the near-field intensity reaches its maximum and minimum value. Both the electric $\left(\lambda_{\text {elec }}=46 \mathrm{~mm}\right)$ and magnetic $\left(\lambda_{\text {mag }}=62 \mathrm{~mm}\right)$ dipolar resonances can be observed. It can be seen how the non-radiative contribution dominates over the radiative part in the near-field regime. However, the latter and the interference term are not negligible as in the case of metallic nanoparticles. ${ }^{2}$
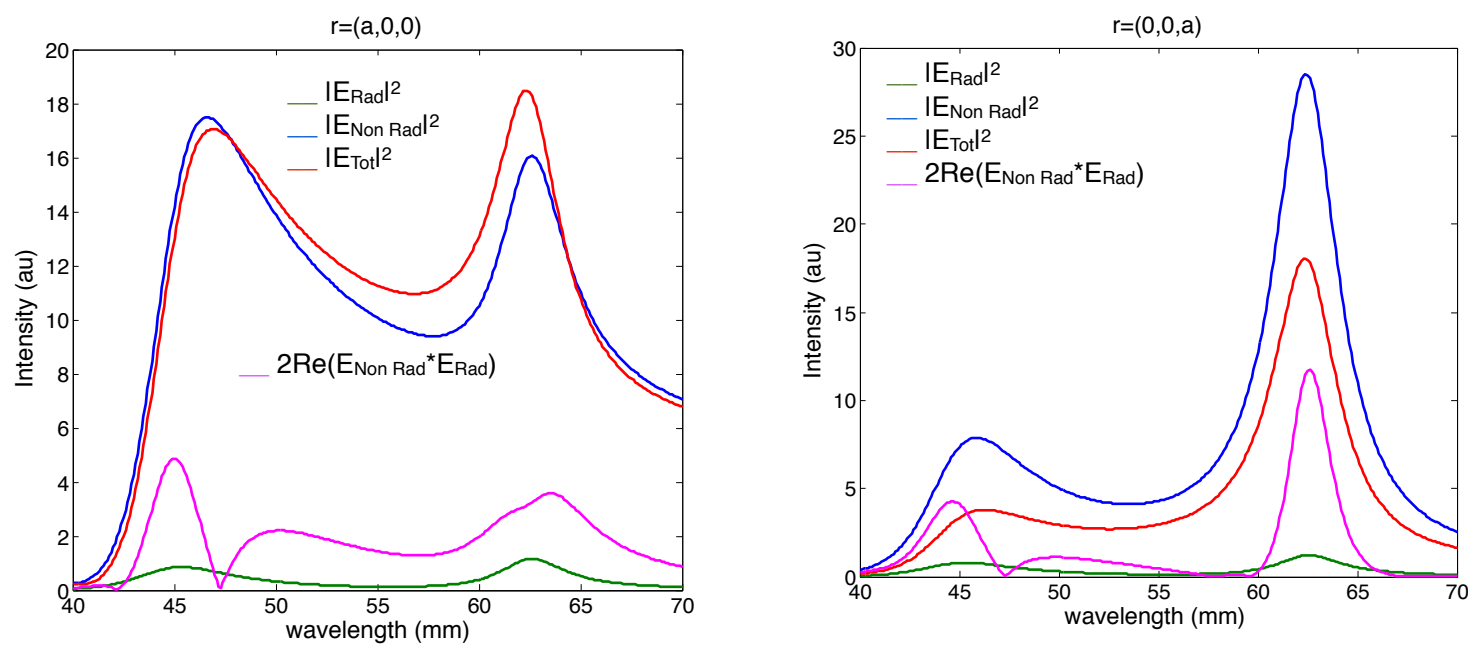

Figure 2. Normalized spectral evolution of $\left|\vec{E}_{s}(\vec{r})\right|^{2}$ (red solid line), $\left|\vec{E}_{s}^{R}(\vec{r})\right|^{2}$ (green solid line), $\left|\vec{E}_{s}^{R}(\vec{r})\right|^{2}$ (blue solid line) and the interference term $2 \operatorname{Re}\left(\vec{E}_{s}^{N-R}(\vec{r})^{*} \cdot \vec{E}_{s}^{R}(\vec{r})\right)$ (magenta solid line) of a magnetodielectric particle with $a=7.5 \mathrm{~mm}$ and $\epsilon=16$ at the positions $\vec{r}=(a, 0,0)$ and $\vec{r}=(0,0, a)$.

By decomposing the scattered field into the electric and magnetic dipolar terms, it is possible to see how the total and non-radiative contributions are red-shifted and broadened with respect to the far-field response (see Fig. 3 and 4). In the case of the magnetic dipole, the red-shift and the broadening are smaller than for the electric dipolar term. By taking this into account, along with the fact that in the near-field the non-radiative contribution dominates over the radiative part, the evanescent waves may be responsible for this phenomenon.

An universal formulation of this phenomenon can be achieved by removing the dependence with the particle parameters (morphology and optical properties) by normalizing to the Mie scattering coefficients in the eqs. (21) and (22). When we are treating the electric dipolar term, the normalization must be to $a_{1}$. However, when the magnetic dipolar term is being studied, the $b_{1}$ coefficient should be used for the normalization.

We have performed a fitting of $\left|\vec{E}_{s}(\vec{r}) / a_{1}\right|^{2}$ and $\left|\vec{E}_{s}^{N-R}(\vec{r}) / a_{1}\right|^{2}$ for the electric dipolar term as a function of the incident wavelength, $\lambda$, to a power law $\left(a+b \lambda^{n}\right)$ (see Fig. 5). At the point $\vec{r}=(a, 0,0)$, both magnitudes scale to a power law with $n \approx 5.3$. However, at the at the point $\vec{r}=(0,0, a),\left|\vec{E}_{s}(\vec{r}) / a_{1}\right|^{2}$ scales with $n \approx 6.3$, and $\left|\vec{E}_{s}^{N-R}(\vec{r}) / a_{1}\right|^{2}$ with $n \approx 5.7$.

The same analysis has been done to the electric field scattered by the magnetic dipolar term (see Fig. 6). In this case, the fitting has been done of the magnitudes $\left|\vec{E}_{s}(\vec{r}) / b_{1}\right|^{2}$ and $\left|\vec{E}_{s}^{N-R}(\vec{r}) / b_{1}\right|^{2}$. On the one hand, at the point $\vec{r}=(a, 0,0)$, both magnitudes scales to a power law with $n \approx 3.5$. On the other, at the point $\vec{r}=(0,0, a)$, $\left|\vec{E}_{s}(\vec{r}) / b_{1}\right|^{2}$ follows a power law with $n \approx 3.0$, whereas $\left|\vec{E}_{s}^{N-R}(\vec{r}) / b_{1}\right|^{2}$ scales with $n \approx 3.5$. 

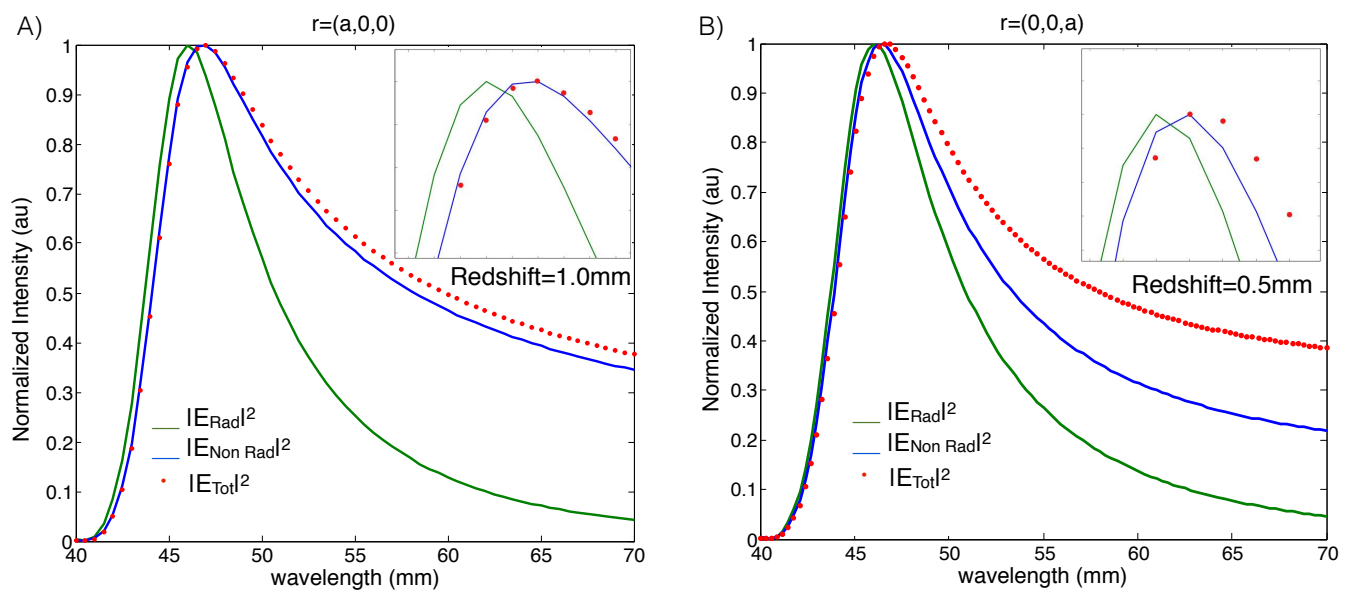

Figure 3. Normalized spectral evolution of $\left|\vec{E}_{s}(\vec{r})\right|^{2}$ (red dots), $\left|\vec{E}_{s}^{R}(\vec{r})\right|^{2}$ (green solid line) and $\left|\vec{E}_{s}^{N-R}(\vec{r})\right|^{2}$ (blue solid line) at the positions: A) $\vec{r}=(a, 0,0)$ and B) $\vec{r}=(0,0, a)$. The electric field is considered to be scattered by the electric dipolar term.

\section{MAGNETIC DIPOLAR TERM}
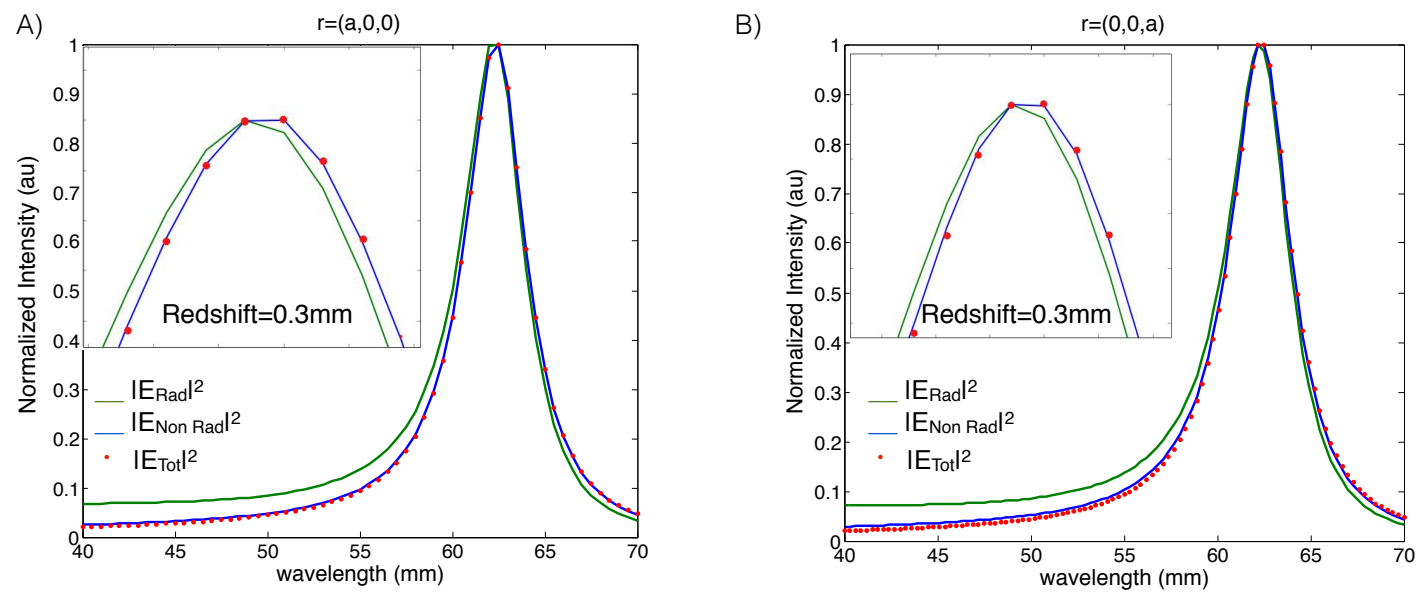

Figure 4. Normalized spectral evolution of $\left|\vec{E}_{s}(\vec{r})\right|^{2}$ (red dots), $\left|\vec{E}_{s}^{R}(\vec{r})\right|^{2}$ (green solid line) and $\left|\vec{E}_{s}^{N-R}(\vec{r})\right|^{2}$ (blue solid line) at the positions: A) $\vec{r}=(a, 0,0)$ and B) $\vec{r}=(0,0, a)$. The electric field is considered to be scattered by the magnetic dipolar term.

These values of $n$ can be compared with the behaviour in the far-field, which is dominated by the radiative contribution. In this latter case, it scales at $\lambda^{2}$ as typical far-field magnitudes like the scattering or extinction efficiencies. However, in the near-field regime, the dominant contribution scales faster $(n>2)$. In addition, the radiative contribution in the near-field, $\left|\vec{E}_{s}^{R}(\vec{r}) / a_{1}\right|^{2}$ and $\left|\vec{E}_{s}^{R}(\vec{r}) / b_{1}\right|^{2}$, is almost independent of the of the wavelength.

The same analysis has been done with the magnetic field scattered by a nanoparticle with the same size and optical properties. The results show the same behaviour as the electric field: a red-shift and broadening of the LSPR peaks in the transition from far- to the near-field regime. 


\section{ELECTRIC DIPOLAR TERM}
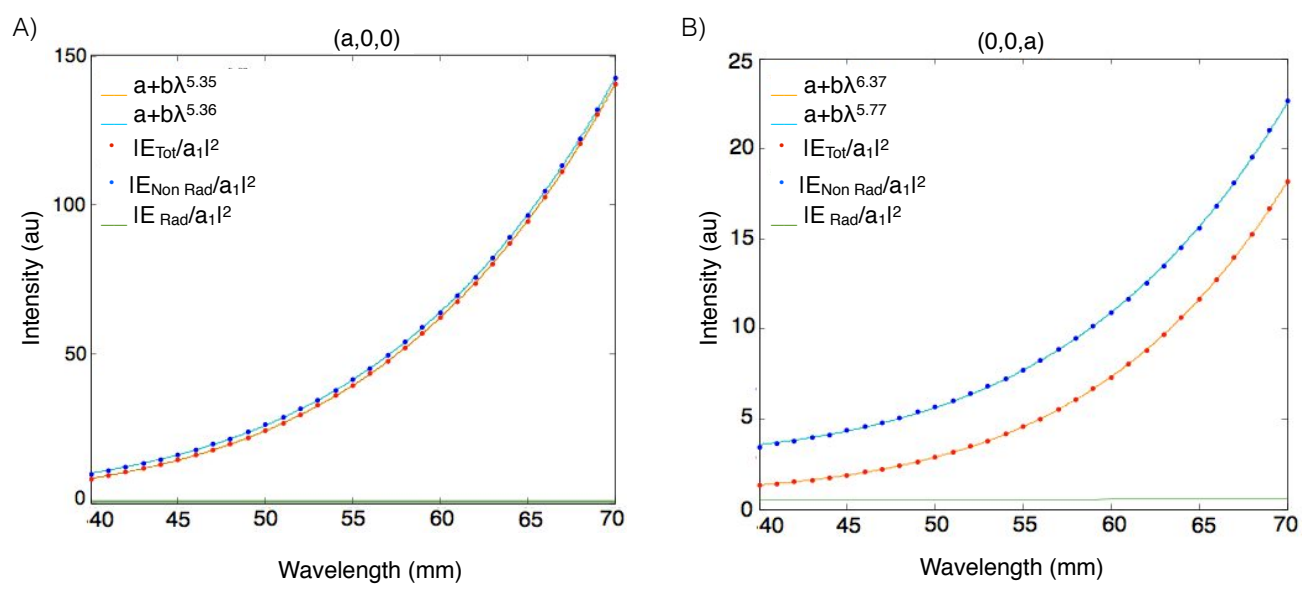

Figure 5. Spectral evolution of $\left|\vec{E}_{s}(\vec{r}) / a_{1}\right|^{2}$ (red dots), $\left|\vec{E}_{s}^{R}(\vec{r}) / a_{1}\right|^{2}$ (blue dots) and $\left|\vec{E}_{s}^{N-R}(\vec{r}) / a_{1}\right|^{2}$ (green line) at two positions: A) $\vec{r}=(a, 0,0)$ and $\mathrm{B}) \vec{r}=(a, 0,0)$. The orange and the fair blue solid lines represent the numeral fitting of the spectral evolution of the total and non radiative contribution to a power law $\left(a+b \lambda^{n}\right)$.

\section{MAGNETIC DIPOLAR TERM}

A)

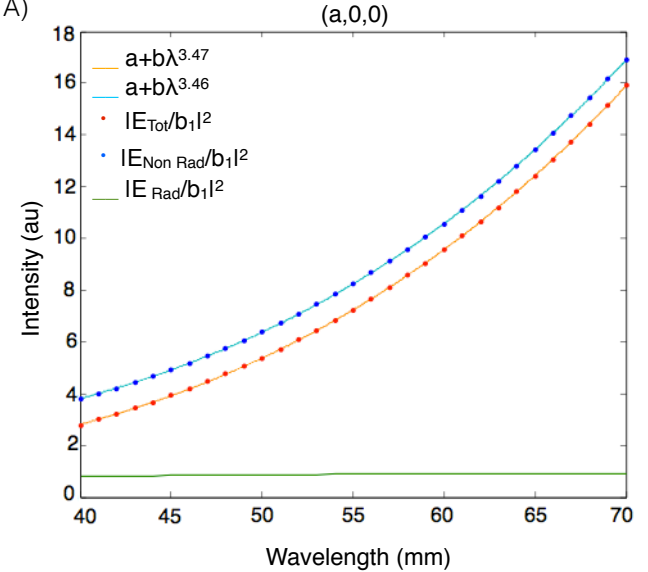

B)

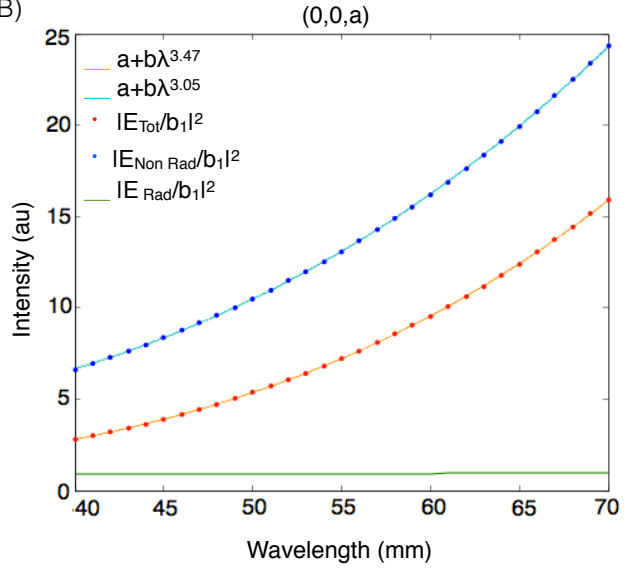

Figure 6. Spectral evolution of $\left|\vec{E}_{s}(\vec{r}) / b_{1}\right|^{2}$ (red dots), $\left|\vec{E}_{s}^{R}(\vec{r}) / b_{1}\right|^{2}$ (blue $\operatorname{dots}$ ) and $\left|\vec{E}_{s}^{N-R}(\vec{r}) / b_{1}\right|^{2}$ (green line) at two positions: A) $\vec{r}=(a, 0,0)$ and $\mathrm{B}) \vec{r}=(a, 0,0)$. The orange and the fair blue solid lines represent the numeral fitting of the spectral evolution of the total and non radiative contribution to a power law $\left(a+b \lambda^{n}\right)$.

\section{CONCLUSIONS}

In this research, we have shown that, as for metallic nanoantennas, in the transition from the far- to the nearfield the resonance peaks of dielectric nano-antennas undergo a red-shift and a broadening. This work points out that this phenomena is caused by the fact that the evanescent waves take the lead role in the near-field regime over the radiative waves, which dominate in the far-field regime. Furthermore, the results for dielectric nano-antennas present several differences from the metallic ones. First of all, magnetodielectric particles present both electric and magnetic resonances. The magnetic one hardly suffers from either a red-shift or broadening, so far-field magnitudes such as the scattering or extinction efficiencies can be used to predict its spectral position. On the contrary, the red-shift and broadening of the electric dipolar resonance is noticeable. In fact, the general 
law $\left(a+b \lambda^{n}\right)$ followed by $\left|\vec{E}_{s}(\vec{r}) / a_{1}\right|^{2}$ and $\left|\vec{E}_{s}^{N-R}(\vec{r}) / a_{1}\right|^{2}$ at $\vec{r}=(a, 0,0)$ (hot spot) is different for metals and dielectrics nanoparticles. Whereas for metals it scales with $n \approx 6$, magnetodielectric materials follows a power law with $n \approx 5$.

We think that the results of the present research can be practical to interpret and predict accurately the spectral positions of the resonance when dielectric nanoantennas are used for spectroscopic techniques, such as SERS, which, on the contrary to what happens with metallic nanosystems, field enhencements and their corresponding photothermal effects cannot destroy or even alter the sample.

\section{ACKNOWLEDGMENTS}

This research was supported by MICINN (Spanish Ministry of Science and Innovation) with project FIS201345854-P. Y. Gutiérrez, and Á. I. Barreda want to express their gratitude to the University of Cantabria for their FPU grants.

\section{REFERENCES}

[1] Zuloaga, J. and Nordlander, P., "On the energy shift between near-field and far-field peak intensities in localized plasmon systems," Nano Letters 11(3), 1280-1283 (2011).

[2] Moreno, F., Albella, P., and Nieto-Vesperinas, M., "Analysis of the Spectral Behavior of Localized Plasmon Resonances in the Near- and Far-Field Regimes," Langmuir 29(22), 6715-6721 (2013).

[3] Alonso-González, P., Albella, P., Neubrech, F., Huck, C., Chen, J., Golmar, F., Casanova, F., Hueso, L. E., Pucci, A., Aizpurua, J., and Hillenbrand, R., "Experimental verification of the spectral shift between nearand far-field peak intensities of plasmonic infrared nanoantennas," Physical Review Letters 110(20), 1-6 (2013).

[4] Yuffa, A. J., Gutierrez, Y., Sanz, J. M., Alcaraz de la Osa, R., Saiz, J. M., González, F., Moreno, F., and Videen, G., "Frequency shift between near- and far-field scattering resonances in dielectric particles," Journal of the Optical Society of America A 32(9), 1638 (2015).

[5] Albella, P., Poyli, M. A., Schmidt, M. K., Maier, S. A., Moreno, F., Sáenz, J. J., and Aizpurua, J., "Low-loss electric and magnetic field-enhanced spectroscopy with subwavelength silicon dimers," Journal of Physical Chemistry C 117(26), 13573-13584 (2013).

[6] Albella, P., Alcaraz de la Osa, R., Moreno, F., and Maier, S. A., "Electric and Magnetic Field Enhancement with Ultralow Heat Radiation Dielectric Nanoantennas: Considerations for Surface-Enhanced Spectroscopies," ACS Photonics 1(6), 524-529 (2014).

[7] Caldarola, M., Albella, P., Cortés, E., Rahmani, M., Roschuk, T., Grinblat, G., Oulton, R. F., Bragas, A. V., and Maier, S. A., "Non-plasmonic nanoantennas for surface enhanced spectroscopies with ultra-low heat conversion," Nature Communications 6, 7915 (2015).

[8] Baffou, G. and Quidant, R., "Thermo-plasmonics: Using metallic nanostructures as nano-sources of heat," Laser and Photonics Reviews 7(2), 171-187 (2013).

[9] García-Etxarri, A., Gómez-Medina, R., Froufe-Pérez, L. S., López, C., Chantada, L., Scheffold, F., Aizpurua, J., Nieto-Vesperinas, M., and Sáenz, J. J., "Strong magnetic response of submicron Silicon particles in the infrared," Optics Express 19(6), 4815 (2011).

[10] Geffrin, J., García-Cámara, B., Gómez-Medina, R., Albella, P., Froufe-Pérez, L., Eyraud, C., Litman, A., Vaillon, R., González, F., Nieto-Vesperinas, M., Sáenz, J., and Moreno, F., "Magnetic and electric coherence in forward- and back-scattered electromagnetic waves by a single dielectric subwavelength sphere," Nature Communications 3, 1171 (2012).

[11] Kvien, K., "Angular spectrum representation of fields diffracted by spherical objects: physical properties and implementations of image field models," Journal of the Optical Society of America A 15(3), 636-651 (1998).

[12] Nieto-Vesperines, M., [Scattering and Diffraction in Physical Optics], World Scientific Publishing, 2 ed. (2006).

[13] Jackson, J. D., [Classical Electrodynamics], John Wiley and Sons, 3 ed. (1998).

[14] Mulholland, G. W., Bohren, C. F., and Fuller, K. A., "Light Scattering by Agglomerates: Coupled Electric and Magnetic Dipole Method," Langmuir 10(8), 2533-2546 (1994). 